\title{
NEOPLASIA TESTICULAR MALIGNA: ANÁLISIS EPIDEMIOLÓGICO DE LOS CASOS NOTIFICADOS EN BRASIL ENTRE 2015 Y 2019
}

\section{ARTÍ́CULO ORIGINAL}

FACCO, Lucas ${ }^{1}$, ALMENDRO, Lucas Pablo², MARQUES, Cristiane Peres ${ }^{3}$, RIBEIRO, Edson Fábio Brito ${ }^{4}$, FECURY, Amanda Alves ${ }^{5}$, DENDASCK, Carla Viana ${ }^{6}$, ARAÚJO, Maria Helena Mendonça de ${ }^{7}$, OLIVEIRA, Euzébio de ${ }^{8}$, DIAS, Claudio Alberto Gellis de Mattos $^{9}$

FACCO, Lucas. Et al. Neoplasia testicular maligna: análisis epidemiológico de los casos notificados en Brasil entre 2015 y 2019. Revista Científica Multidisciplinar Núcleo do Conhecimento. Año. 06, Ed. 10, Vol. 07, págs. 62-74. Octubre de 2021. ISSN: 2448-0959, Enlace de acceso:

https://www.nucleodoconhecimento.com.br/salud/neoplasia-testicular, DOI: 10.32749/nucleodoconhecimento.com.br/salud/neoplasia-testicular

\section{RESUMEN}

La neoplasia testicular es una patología maligna relativamente poco frecuente, representando el $0,5 \%$ de todas las neoplasias masculinas, siendo más frecuente entre los individuos de 15 a 34 años. La presentación clínica más común es la masa testicular o la hinchazón escrotal con o sin dolor o trauma asociado, y el diagnóstico

\footnotetext{
${ }^{1}$ Estudiante del Curso de Medicina de la Universidad Federal de Amapá (UNIFAP).

${ }^{2}$ Estudiante del Curso de Medicina de la Universidad Federal de Acre (UFAC).

${ }^{3}$ Estudiante del Curso de Ingeniería de Producción de la Universidad Federal de Mato Grosso do Sul (UFMS).

${ }^{4}$ Estudiante del Curso de Medicina de la Universidad Federal de Amapá (UNIFAP).

${ }^{5}$ Biomédico, Doctor en Enfermedades Tropicales, Profesor e investigador del Curso Médico de la Universidad Federal de Amapá (UNIFAP).

${ }^{6}$ Teóloga, Doctora en Psicoanálisis Clínica. Trabaja desde hace 15 años con Metodología Científica (Método de Investigación) en Orientación de Producción Científica para Estudiantes de Maestría y Doctorado. Especialista en Investigación de Mercados e Investigación en Salud, Estudiante de Doctorado en Comunicación y Semiótica (PUC SP).

${ }^{7}$ Doctor, Máster en Docencia y Ciencias de la Salud, Profesor e investigador del Curso Médico del Campus Macapá, Universidad Federal de Amapá (UNIFAP).

${ }^{8}$ Biólogo, Doctor en Enfermedades Tropicales, Profesor e investigador del Curso de Educación Física de la Universidad Federal de Pará (UFPA).

${ }^{9}$ Biólogo, Doctor en Teoría e Investigación del Comportamiento, Profesor e investigador del Programa de Postgrado en Educación Profesional y Tecnológica (PROFEPT), Instituto Federal de Amapá (FIPA).

RC: 99986
}

Enlace de acceso: https://www.nucleodoconhecimento.com.br/salud/neoplasia-testicular 
confirmatorio estándar es la orquiectomía. Este estudio tuvo como objetivo analizar epidemiológicamente los casos reportados de neoplasia testicular maligna en Brasil entre 2015 y 2019. Los datos para la investigación epidemiológica se obtuvieron de DATASUS y la investigación bibliográfica se realizó a través de artículos científicos. A partir de la información revelada en esta investigación, es posible observar un diagnóstico creciente de neoplasia maligna de los testículos en Brasil, con casi el doble del número de casos observados entre los años 2015 y 2019. Aunque es relativamente poco común, el cáncer testicular es potencialmente mortal y su gravedad no debe subestimarse y debe diagnosticarse y tratarse lo antes posible. Tiene una alta probabilidad de curación, con tratamiento quirúrgico definitivo, después de un diagnóstico confirmatorio, en la mayoría de los casos, permitiendo que el individuo afectado tenga una vida normal. Por lo tanto, se necesitan más estudios para revelar las razones del aumento de los casos de cáncer testicular en Brasil y en todo el mundo, para comprender si las diferencias regionales están relacionadas con el número de casos o si se trata de una falla en el diagnóstico y registro, además de servir como base para las acciones del gobierno, con el fin de planificar y ejecutar políticas destinadas a combatir los factores desencadenantes de esta enfermedad.

Palabras clave: Neoplasias malignas, Tumores urológicos, Cáncer testicular, Orquiectomía inguinal, Epidemiología.

\section{INTRODUCCIÓN}

El cáncer o neoplasia se produce por la proliferación de células del organismo que presentan cambios morfológicos y funcionales y provocan trastornos tisulares. Tales cambios pueden tener causas genéticas o ambientales (Dias et al., 2017).

La neoplasia testicular es una patología maligna relativamente poco frecuente, que representa el $0,5 \%$ de todas las neoplasias masculinas y el $5 \%$ de los tumores urológicos (Rosen et al., 2011; Nci, 2021), y es más frecuente entre individuos de 15 a 34 años (Baird et al., 2018). Además, la variante más frecuente fue el tumor de células germinales, con un $95 \%$ de los casos, y el diagnóstico más frecuente en masas 
palpables en estadio I clínicamente localizado (Adra y Einhorn, 2017; Pierorazio et al., 2018).

Se señalaron factores de riesgo para el desarrollo de este tipo de cáncer: antecedentes previos, con un riesgo de 5 a $6 \%$ de superación del testículo contralateral; antecedentes familiares, con un riesgo de 8 a 10 veces entre hermanos y de 4 a 6 veces entre hijos de un portador; criptorquidia, con odds ratio (OR) 4,3, intervalo de confianza del 95\%: 3,6-5,1; orquidopexia tardía (fijación testicular en el tallo escrotal) con OR de 5,8 en comparación con la temprana; y síndrome de Klinefelter (Hemminki y Li, 2004; Walsh et al., 2007; Cook et al., 2010; Lip et al., 2012; Chan et al., 2014; Kier et al., 2016; Nery, 2019). La presentación clínica más común es la masa testicular o la hinchazón escrotal con o sin dolor/trauma asociado, teniendo como diagnóstico diferencial la orquitis o la epididimitis, pueden iniciar el tratamiento con antibióticos (Nery, 2019). Las metástasis pueden ocurrir, dependiendo de la ubicación, tales como: síntomas gastrointestinales; ginecomastia; dolor de cabeza; dolor lumbar; masa del cuello; síntomas (disnea, tos y hemoptisis) (Shaw, 2008).

La metodología diagnóstica para dicha neoplasia comienza durante el examen físico, con la palpación del tallo escrotal, pero esto genera resultados ambiguos, por lo que el uso de la investigación radiológica con ecografía transescrotal, en la que se ha observado el uso creciente en la detección de lesiones impalpables o ambiguas (Dieckmann et al., 2013; Cheng et al., 2018). El diagnóstico confirmatorio estándar es la orquiectomía radical (cirugía para extirpar uno o ambos testículos y todo el cordón espermático), que permite establecer el carácter y a menudo ya se usa con el tratamiento (Ghoreifi y Djaladat, 2019). El tratamiento estándar de oro para masas testiculares con sospecha de malignidad, sin signos de metástasis, se estableció como orquiectomía radical con extirpación hasta el nivel del anillo inguinal interno, que a menudo se realiza durante un procedimiento de diagnóstico y ofrece la posibilidad de reemplazo por prótesis testicular (Krege et al., 2008; Robinson et al., 2015; Ghoreifi e Djaladat, 2019). Sin embargo, los marcadores tumorales séricos (alfa-fetoproteína AFP - y beta-gonadotrofia caónica humana - Beta-HCG ) se utilizan para ayudar a evaluar la efectividad del tratamiento y evaluar el pronóstico para el paciente, ya que 
deben establecerse antes y después del tratamiento, así como durante el período de vigilancia (Gilligan et al., 2019).

En el contexto global, hubo 72,000 diagnósticos y 9,000 muertes por año para este proceso neoplásico, y el riesgo de que un hombre desarrolle cáncer testicular durante algún momento de su vida se estimó en 1 de cada 250 hombres (Fitzmaurice et al., 2017; Acs, 2021). La proyección estimada para 2021 de nuevos casos de cáncer testicular en los Estados Unidos de América fue de 9,470 diagnósticos y 440 muertes, además de una mayor frecuencia entre los individuos blancos (6.9 individuos afectados por 100,000 hombres) en comparación con los afroamericanos (1.2 individuos afectados por 100,000 hombres) (Ghazarian et al., 2014; Acs, 2021).

A su vez, a nivel nacional, hubo cierta escasez de datos epidemiológicos sobre esta neoplasia, pero hubo un aumento de la mortalidad en comparación con los datos de 2015 (359 muertes, que representan el 0,05\% de la mortalidad general) y 2019 (446 muertes, que representan el 0,06\% de la mortalidad general) del Atlas de Mortalidad por Cáncer - Sistema de Información de Mortalidad (Nery, 2019; Inca, 2021).

\section{GOL}

Analizar epidemiológicamente los casos reportados de neoplasia testicular maligna en Brasil entre 2015 y 2019.

\section{MÉTODO}

La investigación utilizó metodología cuali-cuantitativo (método mixto), con datos gubernamentales, ya que además de los datos numéricos implicaba la interpretación de fenómenos (Facco et al., 2021).

La recolección de datos se realizó en la base de datos DATASUS (http://datasus.saude.gov.br/). Los datos nacionales se recopilaron de acuerdo con los siguientes pasos: A) Se accedió al enlace datasus.saude.gov.br, se deslizó la flecha con el mouse a la pestaña "Servicios destacados", inmediatamente después de seleccionar la opción "TABNET"; B) En la página siguiente "TABNET" se hizo clic en RC: 99986

Enlace de acceso: https://www.nucleodoconhecimento.com.br/salud/neoplasia-testicular 
la opción "Epidemiología y Morbilidad" y por lo tanto se seleccionó la opción "Tiempo hasta el inicio del tratamiento del cáncer - PANEL - oncología". A partir de ahí, se siguieron los pasos: A) En la casilla "Línea", se seleccionó "Año de diagnóstico" durante todo el proceso; B) En el recuadro "Medidas", se seleccionaron "Casos" a lo largo del proceso; C) Y en el recuadro "Columna" se seleccionaron los siguientes: "Estadificación", "Grupo de Edad", "Modalidad Terapéutica", "Tiempo de Tratamiento", "UF de Residencia", "Uf de Diagnóstico" y "UF de Tratamiento". Todos los datos recopilados en el sistema cubren los períodos 2015 a 2019. D) En el recuadro "selección disponible" de la opción "Diagnóstico", se seleccionó la opción "Neoplasias Malignas (Ley № 12.732/12) y en "Diagnóstico Detallado" se seleccionó la opción "Neoplasias malignas C62 de los testículos". En las otras casillas de verificación disponibles, se mantuvieron las opciones estándar del sistema DATASUS. Los datos fueron compilados dentro de la aplicación Excel, un componente de la suite Office de Microsoft Corporation, y los datos de las regiones de Brasil fueron aglutinados a partir de los datos proporcionados por cada uno de los estados de las regiones apropiadas. La investigación bibliográfica se realizó en artículos científicos, utilizando computadoras personales de los autores del presente estudio.

\section{RESULTADOS}

La Figura 1 denota el número de casos de neoplasia maligna de los testículos en Brasil entre 2015 y 2019, a través de la estadificación. Se observa que la estadificación de grado 3 fue la más completa (789 casos), seguida de grado 1 (742), grado 2 (596) y 0 (141). Se observa que, en la gran mayoría de los casos notificados, el criterio de estadificación se clasificó como "no se aplica", además de que 193 casos se notificaron como "ignorados". 
La Figura 1 - Muestra el número de casos de neoplasia maligna de los testículos en Brasil entre 2015 y 2019, a través de la estadificación.

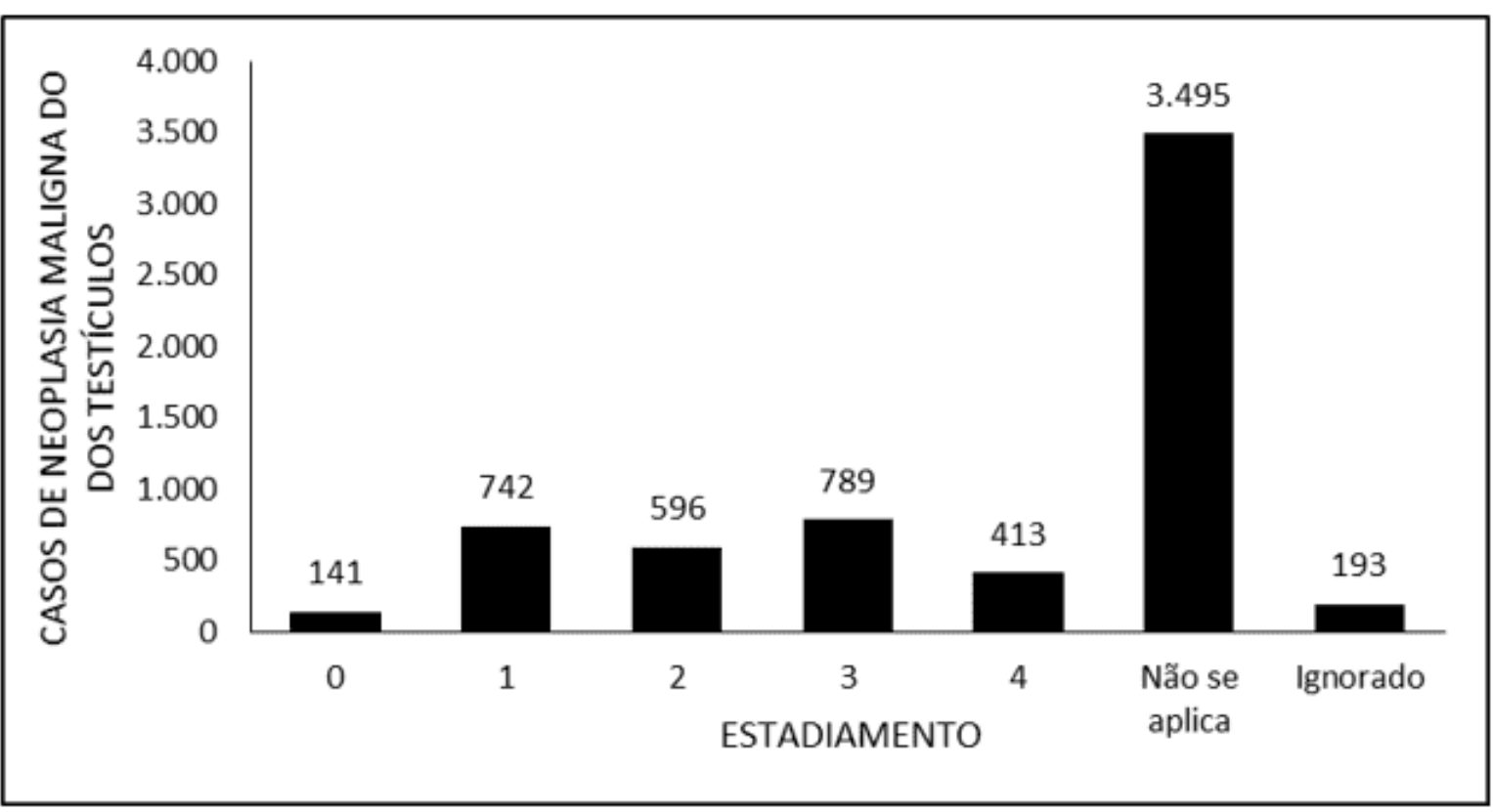

Fuente: autores.

La Tabla 1 denota el número de casos de neoplasia maligna de los testículos en Brasil entre los años 2015 y 2019, según el grupo de edad. Se observa que los 5 rangos de edad con el mayor número de casos reportados de neoplasia maligna testicular son: 25 a 29 años (1,312), 30 a 34 años (1,157), 20 a 24 años (1,072), 35 a 39 años (811) y 0 a 19 años (563). Además, se señaló que el mayor número de casos reportados ocurrió en 2019, con 1,841 casos. 
La Tabla 1 - Muestra el número de casos de neoplasia maligna de los testículos en Brasil entre los años 2015 y 2019, según el grupo de edad.

\begin{tabular}{ccccccc}
\hline Faixa Etária & $\mathbf{2 0 1 5}$ & $\mathbf{2 0 1 6}$ & $\mathbf{2 0 1 7}$ & $\mathbf{2 0 1 8}$ & $\mathbf{2 0 1 9}$ & Total \\
\hline 0 a 19 anos & 81 & 91 & 91 & 125 & 175 & 563 \\
20 a 24 anos & 177 & 206 & 175 & 245 & 269 & 1.072 \\
25 a 29 anos & 225 & 222 & 223 & 315 & 327 & 1.312 \\
30 a 34 anos & 206 & 221 & 206 & 230 & 294 & 1.157 \\
35 a 39 anos & 120 & 121 & 143 & 192 & 235 & 811 \\
40 a 44 anos & 57 & 62 & 80 & 109 & 112 & 420 \\
45 a 49 anos & 33 & 50 & 33 & 68 & 78 & 262 \\
50 a 54 anos & 28 & 22 & 29 & 52 & 56 & 187 \\
55 a 59 anos & 15 & 8 & 15 & 35 & 59 & 132 \\
60 a 64 anos & 8 & 10 & 13 & 32 & 59 & 122 \\
65 a 69 anos & 7 & 8 & 5 & 39 & 51 & 110 \\
70 a 74 anos & 4 & 3 & 3 & 31 & 50 & 91 \\
75 a 79 anos & 4 & 1 & 0 & 19 & 42 & 66 \\
80 anos e mais & 1 & 2 & 2 & 25 & $\mathbf{1 . 8 4 1}$ & $\mathbf{6 . 3 6 9}$ \\
\hline Total & 966 & $\mathbf{1 . 0 2 7}$ & $\mathbf{1 . 0 1 8}$ & $\mathbf{1 . 5 1 7}$ & & 64 \\
\hline
\end{tabular}

Fuente: autores.

La Figura 2 cubre el número de casos de neoplasia maligna de los testículos en Brasil entre 2015 y 2019, a través de la modalidad terapéutica utilizada. Se observa que la mayor proporción de pacientes (3.495) fueron tratados quirúrgicamente, y 2.594 fueron tratados con quimioterapia. La radioterapia se utilizó en 86 pacientes y solo 1 paciente hizo uso de las tres modalidades terapéuticas. No hubo información sobre el tratamiento utilizado en 193 pacientes.

RC: 99986

Enlace de acceso: https://www.nucleodoconhecimento.com.br/salud/neoplasia-testicular 
Figura 2 - Muestra el número de casos de neoplasia maligna de los testículos en Brasil entre 2015 y 2019 , a través de la modalidad terapéutica.

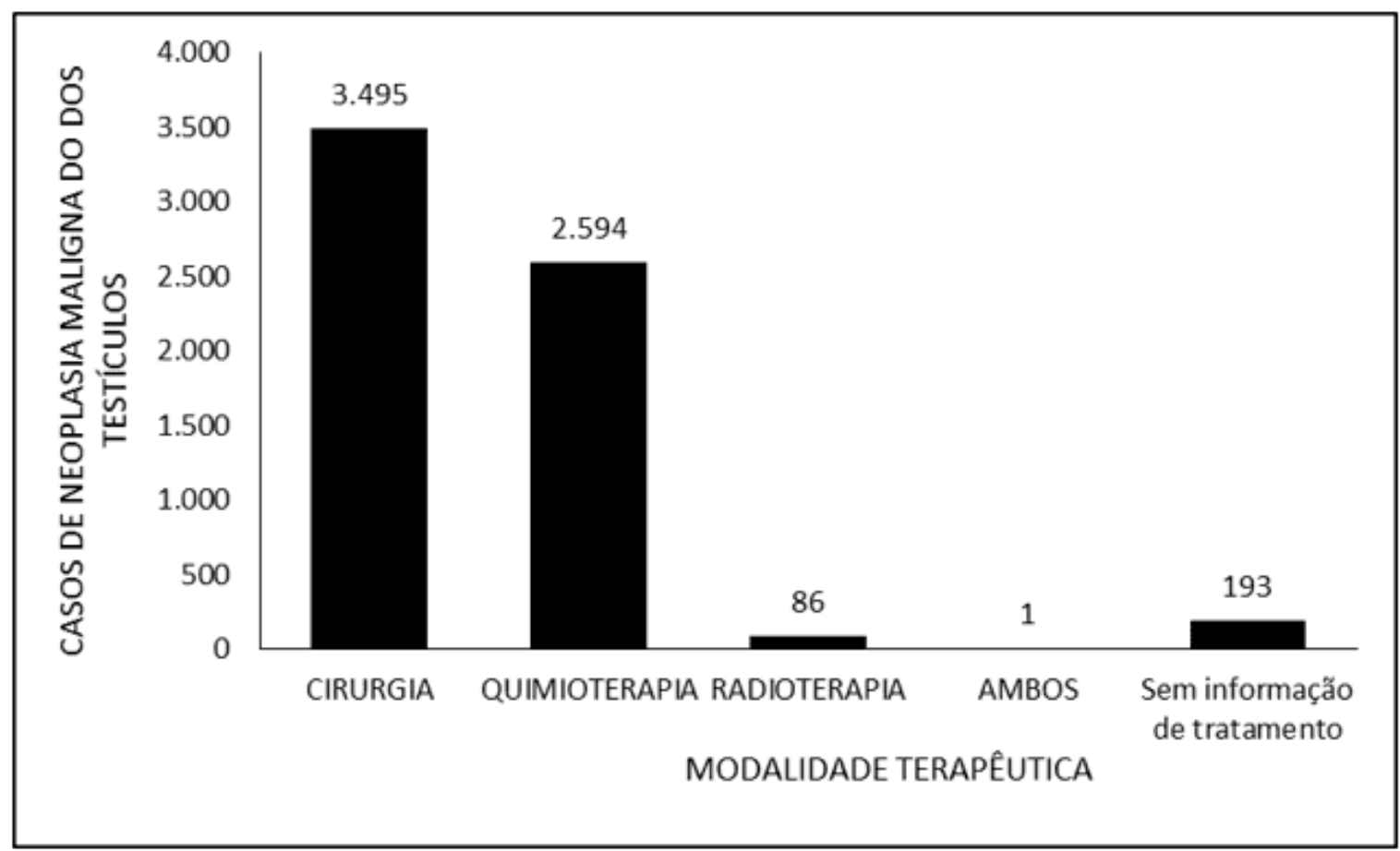

Fuente: autores.

La Figura 3 denota el número de casos de neoplasia maligna de los testículos en Brasil entre 2015 y 2019, a través del momento del tratamiento. La gran mayoría de los casos notificados se refieren a un tiempo de tratamiento de hasta 30 días (4.216), seguido de más de 60 días (1.211) y de 31 a 60 días (749). No hubo información sobre el tiempo de tratamiento para 193 pacientes.

RC: 99986

Enlace de acceso: https://www.nucleodoconhecimento.com.br/salud/neoplasia-testicular 
La Figura 3 - Muestra el número de casos de neoplasia maligna de los testículos en Brasil entre 2015 y 2019, a través del momento del tratamiento.

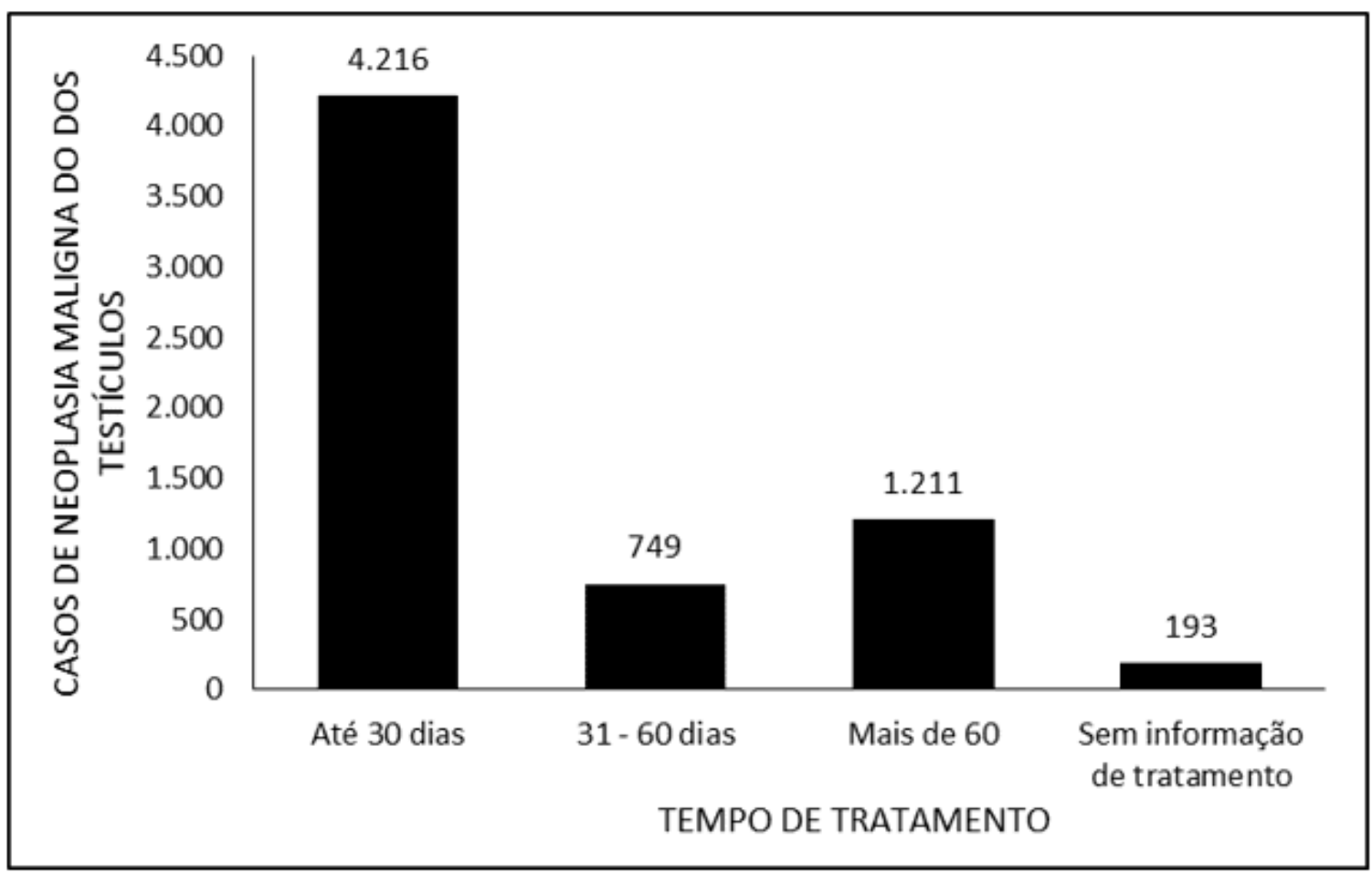

Fuente: autores.

La Figura 4 denota el número de casos de neoplasia maligna de los testículos en Brasil entre 2015 y 2019, a través de la región de residencia. La mayoría de los casos se refieren a las regiones Sureste (2.785), seguidas de las regiones Sur (2.232), Noreste (743), Medio Oeste (374) y Norte (235). 
La Figura 4 - Muestra el número de casos de neoplasia maligna de los testículos en Brasil entre 2015 y 2019, a través de la región de residencia.

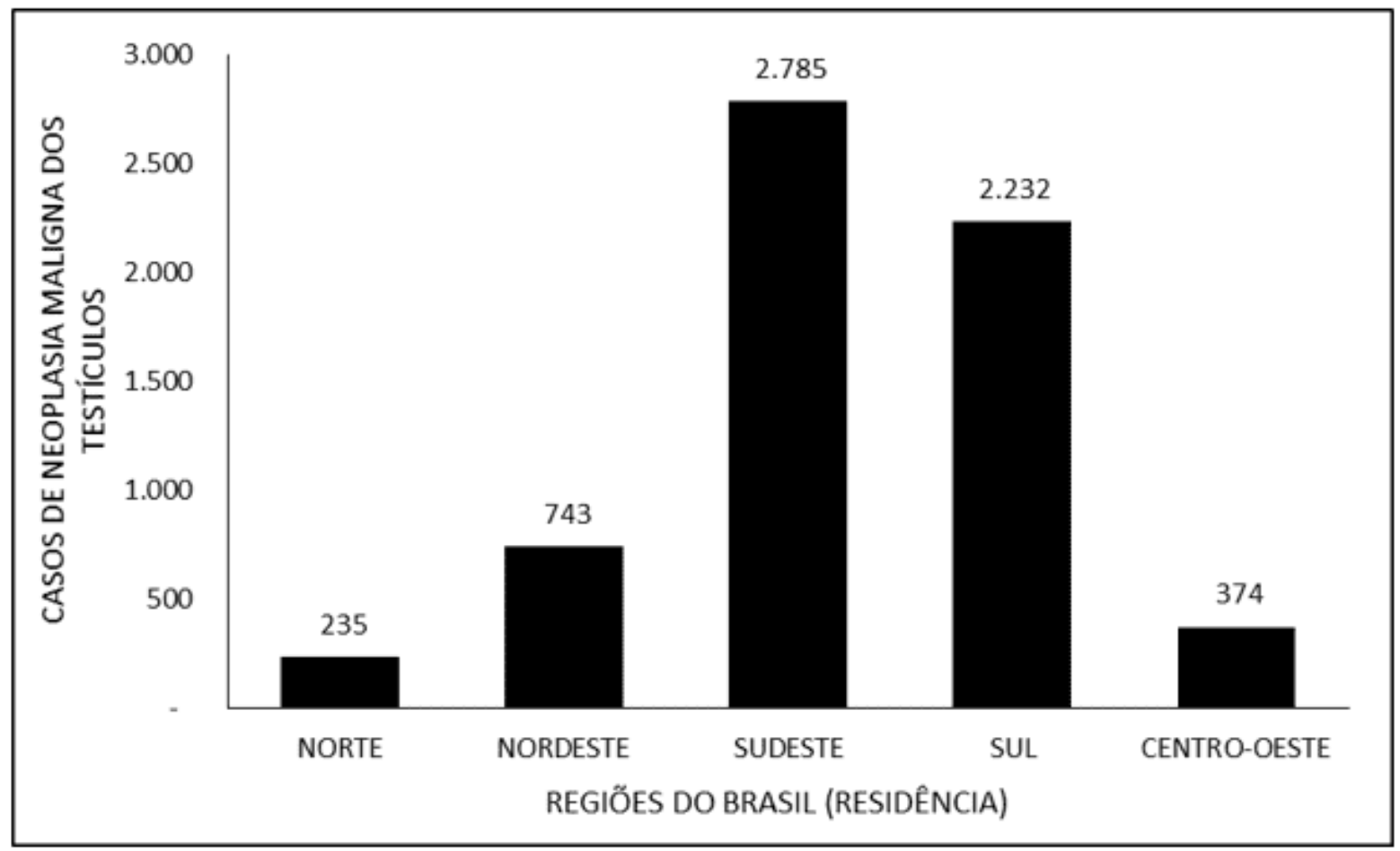

Fuente: autores.

La Figura 5 denota el número de casos de neoplasia maligna de los testículos en Brasil entre 2015 y 2019, a través de la región de diagnóstico. Se observa que la mayor proporción de diagnósticos ocurrió en el Sureste $(2,829)$, seguido por el Sur $(2,245)$, Noreste (731), Medio oeste (333) y Norte (231). 
Figura 5 - Muestra el número de casos de neoplasia maligna de los testículos en Brasil entre 2015 y 2019, a través de la región de diagnóstico.

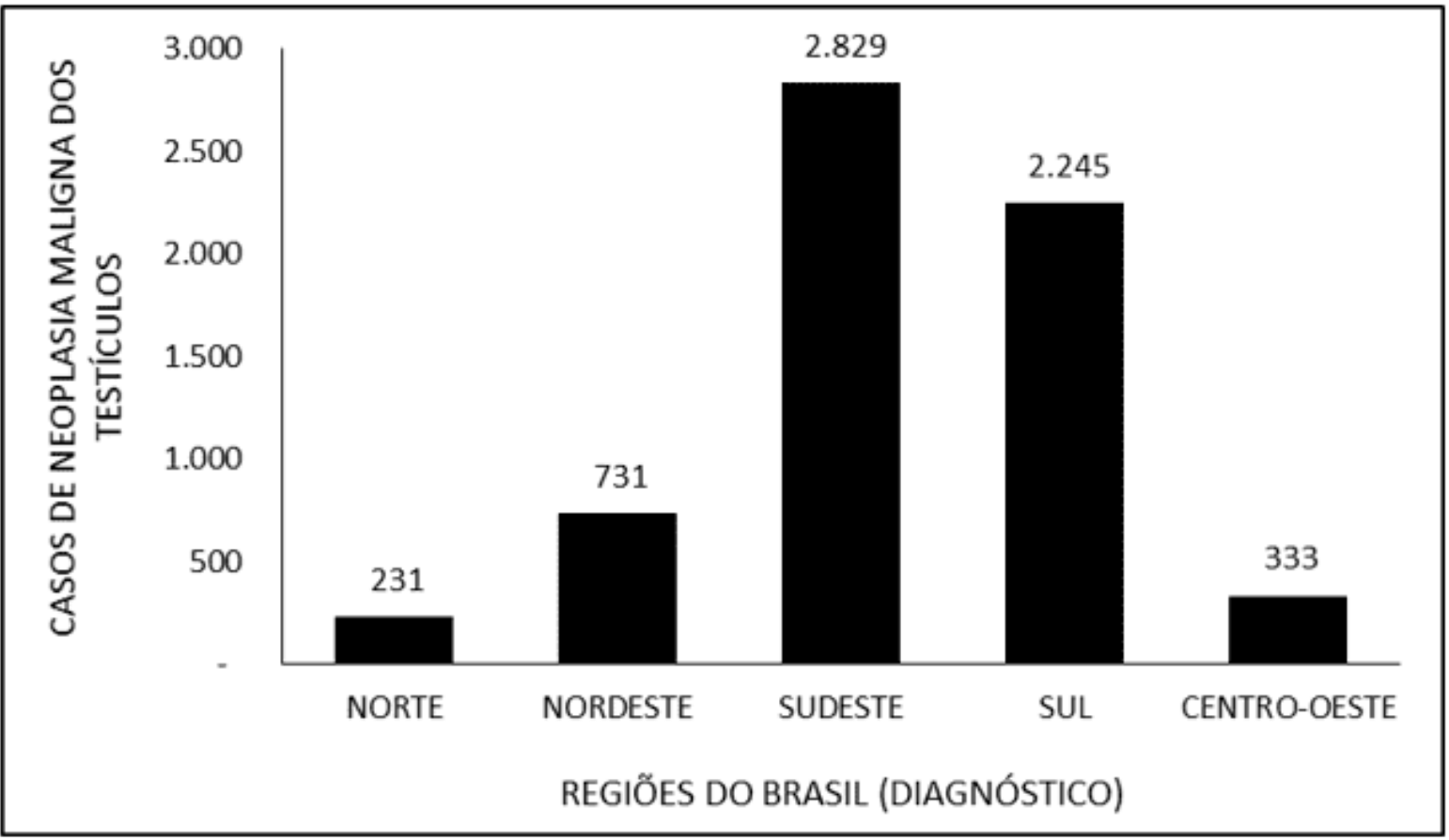

Fuente: autores.

La Figura 6 cubre el número de casos de neoplasia maligna de los testículos en Brasil entre 2015 y 2019, a través de la región de tratamiento. La mayoría de los casos (2.859) fueron reportados como "ignorados". La región Sureste tuvo la mayor notificación de tratamientos para la malignidad testicular, con 2.731 notificaciones, seguida por el Sur (2.211), el Noreste (689), el Medio Oeste (327) y el Norte (218). 
Figura 6 - Muestra el número de casos de neoplasia maligna de los testículos en Brasil entre 2015 y 2019, a través de la región de tratamiento.

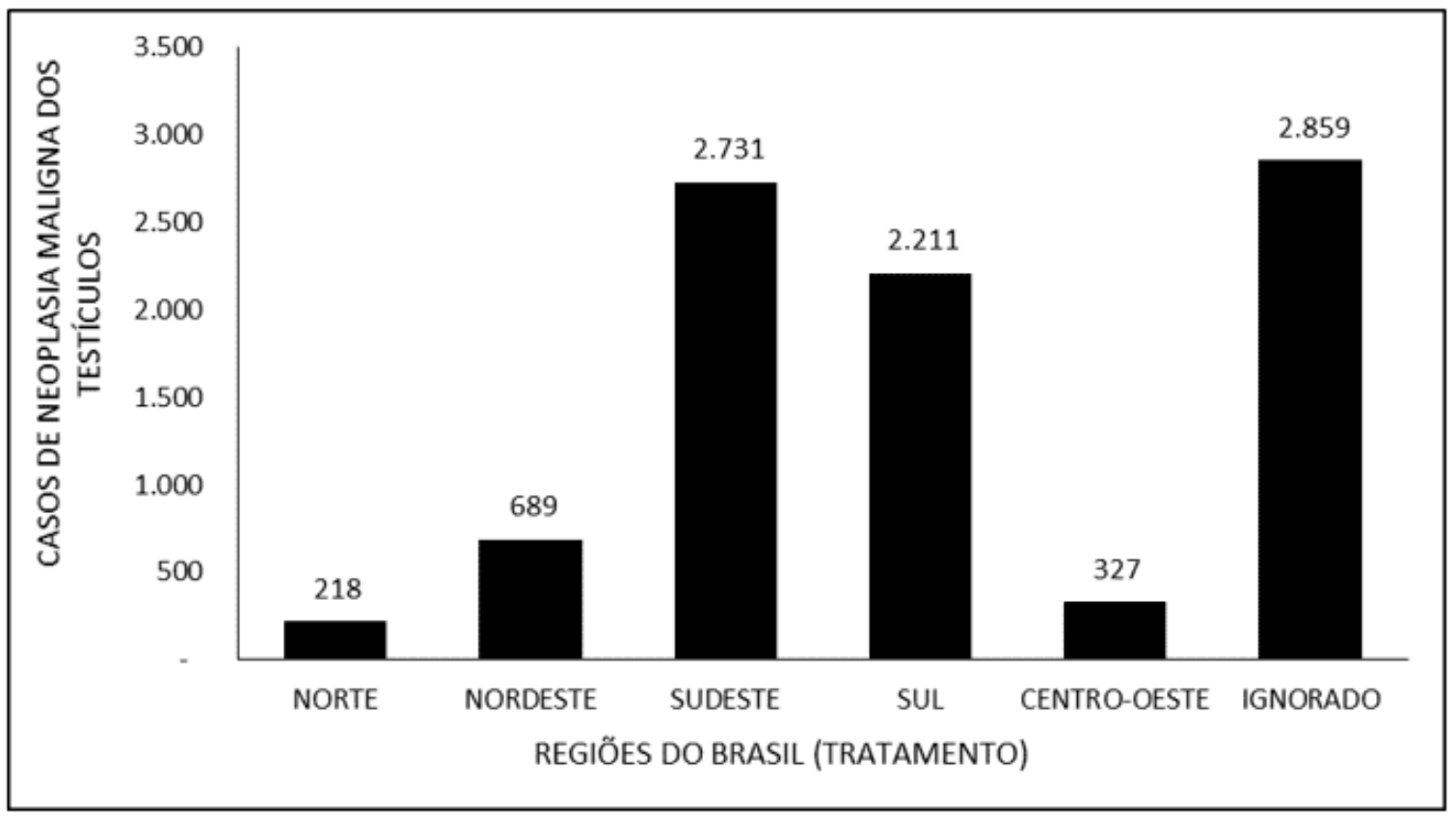

Fuente: autores.

\section{DISCUSIÓN}

Como se puede ver en la Tabla 1, hubo un aumento creciente en el número de casos de neoplasia testicular maligna en Brasil entre 2015 y 2019, siguiendo la tendencia mundial que, durante décadas, ha estado aumentando la tasa de incidencia de cáncer testicular en muchos países. Sin embargo, las razones de este fenómeno aún se desconocen (Acs, 2021).

El elevado número de diagnósticos de neoplasia maligna de los testículos en estadio 3 puede basarse en la evolución silenciosa de una masa sólida indolora, que muchas veces es mal evaluada y diagnosticada como orquitis o epididimitis, que termina siendo tratada de forma inadecuada (benigna), generando un retraso en el abordaje correcto, que puede ser de hasta 20 semanas, permitiendo la aparición de metástasis (Moul, 2007; Shaw, 2008; Nery, 2019). Estos, a su vez, presentan síntomas clínicos, permitiendo así el diagnóstico del proceso neoplásico y metastásico ya presente, como los síntomas respiratorios (disnea, tos y hemoptisis) (Shaw, 2008).

RC: 99986

Enlace de acceso: https://www.nucleodoconhecimento.com.br/salud/neoplasia-testicular 
Como se ha ilustrado en otros estudios, el grupo de edad más común para desarrollar cáncer testicular fue de 15 a 34 años, corroborando así los datos presentados en este estudio (Baird et al., 2018). Además, los factores que pueden haber llevado a la detección de neoplasia en este grupo de edad pueden ser la sexarquia precoz y la vida sexual activa, que permiten un mayor autoconocimiento corporal y múltiples parejas sexuales, lo que permite notar antes la formación de un edema escrotal de causa desconocida y la consiguiente búsqueda de atención médica (Adra y Einhorn, 2017). Es importante mencionar que no se recomienda el tamizaje del cáncer testicular, ya que existe evidencia de que su práctica trae más beneficios que riesgos (Inca, 2021).

El procedimiento quirúrgico se estableció como el tratamiento estándar de oro, ya que elimina el foco neoplásico del cordón espermático al nivel del anillo inguinal inferior, evitando la manipulación excesiva de la cadena ganglionar y el foco neoplásico en sí, evitando así la extravasación celular del proceso canceroso (Krege et al., 2008; Rajpert-De-Meyts et al., 2016; Ghoreifi e Djaladat, 2019; Eau, 2021).

Una justificación para el tratamiento con triple modalidad es la presencia de metástasis a distancia, lo que hace necesario utilizar quimioterapia y radioterapia junto con el procedimiento quirúrgico, aumentando la supervivencia al 96\% en 10 años (Nery, 2019; Nci, 2021), variando las combinaciones y ciclos de quimiorradioterapia según la etapa clínica y variables individuales,como 4 ciclos de BEP (Cisplatino $20 \mathrm{mg} / \mathrm{m} 2$, intravenoso del día 1 al día 5; Etopósido $100 \mathrm{mg} / \mathrm{m}^{2}$,intravenoso del día 1 al día 5; Bleomicina 30 ui intravenosa los días 2, día 9 y día 16; Repetir cada 21 días) (Nery, 2019).

Se entiende que, a menudo, durante el proceso de diagnóstico confirmatorio, se elige la orquiectomía inguinal, que se considera un procedimiento terapéutico, ya que elimina el foco primario de la neoplasia (Ghoreifi y Djaladat, 2019). Además, tenemos que en el momento de la formulación de hipótesis diagnósticas con exámenes radiológicos y elección para orquiectomía radical hasta el nivel del anillo inguinal inferior, y sustitución por prótesis es necesario investigar el riesgo quirúrgico y si 
existen focos metastásicos en otras regiones mediante marcadores tumorales. (Krege et al., 2008; Robinson et al., 2015; Ghoreifi e Djaladat, 2019; Gilligan et al., 2019).

Considerando que el $49,5 \%$ de los hospitales calificados para el manejo de procesos neoplásicos se ubican en la región Sureste del país, seguidos por la región Sur, con un $24,2 \%$, y que ambos tienen mayor disponibilidad de unidades de reconocimiento, es posible inferir que también cuentan con un mejor sistema de reconocimiento diagnóstico de esta neoplasia. Además, las tasas de tratamiento más altas se encuentran en las regiones Sur y Sudeste de Brasil, con el 37,9\% de todos los diagnósticos registrados solo en el estado de São Paulo (Inca, 2019a; 2019b; 2019c; 2019d; 2019e; 2019f; 2019g; 2019h).

El cáncer testicular es una neoplasia maligna que afecta a un número considerable de adultos jóvenes con vida sexual activa y que puede llevar a la muerte (Park et al., 2018). Sin embargo, representa una de las neoplasias malignas más curables cuando se identifica y trata fácilmente con un enfoque multimodal. Con un manejo efectivo, el pronóstico es excelente, con tasa de curación $>90 \%$ y tasa de supervivencia a cinco años> 95\% (Smith et al., 2018).

\section{CONCLUSIÓN}

A partir de la información revelada en esta investigación, es posible observar un diagnóstico creciente de neoplasia maligna de los testículos en Brasil, con casi el doble del número de casos observados entre los años 2015 y 2019. Aunque es relativamente poco común, el cáncer testicular es potencialmente mortal y su gravedad no debe subestimarse y debe diagnosticarse y tratarse lo antes posible. Tiene una alta probabilidad de curación, con tratamiento quirúrgico definitivo, después de un diagnóstico confirmatorio, en la mayoría de los casos, permitiendo que el individuo afectado tenga una vida normal. Por lo tanto, se necesitan más estudios para revelar las razones del aumento de los casos de cáncer testicular en Brasil y en todo el mundo, para comprender si las diferencias regionales están relacionadas con el número de casos o si se trata de una falla en el diagnóstico y registro, además de 
servir como base para las acciones del gobierno, con el fin de planificar y ejecutar políticas destinadas a combatir los factores desencadenantes de esta enfermedad.

\section{REFERENCIAS}

ACS. Key Statistics for Testicular Cancer. New York NY, 2021. Disponível em: < https://www.cancer.org/cancer/testicular-cancer/about/key-statistics.html\#references >. Acesso em: 05 mar 2021.

ADRA, N.; EINHORN, L. H. Testicular Cancer Update. Clinical Advances in Hematology \& Oncology, v. 15, n. 5, p. 386-396, 2017.

BAIRD, D. C.; MEYERES, G.; HU, J. S. Testicular Cancer: Diagnosis and Treatment. American Family Physician, v. 97, n. 4, p. 261-268, 2018.

CHAN, E.; WAYNE, C.; NASR, A. Ideal timing of orchiopexy: a systematic review. Pediatric Surgery International, v. 30, n. 1, p. 87-97, 2014.

CHENG, L. et al. Testicular cancer. Nature Reviews Disease Primers, v. 4, n. 29, p. 1-24, 2018.

COOK, M. B. et al. Systematic review and meta-analysis of perinatal variables in relation to the risk of testicular cancer-experiences of the son. International Journal of Epidemiology, v. 39, n. 6, p. 1605-1618, 2010.

DIAS, A. D. A. et al. Update on the Main Aspects Related to Breast Cancer. Revista Científica Multidisciplinar Núcleo do Conhecimento, v. 4, p. 517, 2017. Disponível em: < https://www.nucleodoconhecimento.com.br/health/breast-cancer >.

DIECKMANN, K. P.; FREY; LOCK, G. Contemporary diagnostic work-up of testicular germ cell tumours. Nat Rev Urol v. 10, p. 703-712, 2013.

EAU. Testicular Cancer. Düsseldorf DE, 2021. Disponível em: < https://uroweb.org/guideline/testicular-cancer/\#1 >. Acesso em: 06 mar 2021.

RC: 99986

Enlace de acceso: https://www.nucleodoconhecimento.com.br/salud/neoplasia-testicular 
FACCO, L. et al. Neoplasia maligna de esôfago: uma análise epidemiológica dos casos notificados no Brasil entre 2015 e 2019. Research, Society and Development, v. $10, \quad$ n. $2, \quad$ p. 1-14, 2021. Disponível em: < https://rsdjournal.org/index.php/rsd/article/view/12750/11622 >.

FITZMAURICE, C. et al. Global, Regional, and National Cancer Incidence, Mortality, Years of Life Lost, Years Lived With Disability, and Disability-Adjusted Life-years for 32 Cancer Groups, 1990 to 2015: A Systematic Analysis for the Global Burden of Disease Study. .JAMA Oncol, v. 3, n. 4, p. 524-548, 2017.

GHAZARIAN, A. A. et al. Recent trends in the incidence of testicular germ cell tumors in the United States. Andrology, v. 3, n. 1, p. 13-18, 2014.

GHOREIFI, A.; DJALADAT, H. Management of Primary Testicular Tumor. Urologic Clinics of North America, v. 46, n. 3, p. 333-339, 2019.

GILLIGAN, T. et al. Testicular Cancer, Version 2.2020, NCCN Clinical Practice Guidelines in Oncology. Journal of the National Comprehensive Cancer Network, v. 17, n. 12, p. 1529-1554, 2019.

HEMMINKI, K.; LI, X. Familial risk in testicular cancer as a clue to a heritable and environmental aetiology. British Journal of Cancer, v. 90, n. 9, p. 1765-1770, 2004.

INCA. Onde tratar pelo SUS. Brasília DF, 2019a. Disponível em: < https://www.inca.gov.br/onde-tratar-pelo-

sus\#: :text=Existem\%20atualmente\%20317\%20unidades\%20e,exame\%20até\%20ci rurgias\%20mais\%20complexas >. Acesso em: 06 mar 2021.

. Paraná. Brasília DF, 2019b. Disponível em: < https://www.inca.gov.br/ondetratar-pelo-sus/parana >. Acesso em: 06 mar 2021.

Santa Catarina. Brasília DF, 2019c. Disponível em: < https://www.inca.gov.br/onde-tratar-pelo-sus/santa-catarina $>$.

RC: 99986

Enlace de acceso: https://www.nucleodoconhecimento.com.br/salud/neoplasia-testicular 
- Rio Grande do Sul. Brasília DF, 2019d. Disponível em: < https://www.inca.gov.br/onde-tratar-pelo-sus/rio-grande-sul >. Acesso em: 06 mar 2021.

Minas Gerais. Brasília DF, 2019e. Disponível em: < https://www.inca.gov.br/onde-tratar-pelo-sus/minas-gerais >. Acesso em: 12 jan 2021.

São Paulo. Brasília DF, 2019f. Disponível em: < https://www.inca.gov.br/onde-tratar-pelo-sus/sao-paulo >. Acesso em: 15 jan 2021.

Rio de Janeiro. Brasília DF, 2019g. Disponível em: < https://www.inca.gov.br/onde-tratar-pelo-sus/rio-janeiro >. Acesso em: 15 jan 2021.

Espírito Santo. Brasília DF, 2019h. Disponível em: < https://www.inca.gov.br/onde-tratar-pelo-sus/espirito-santo >. Acesso em: 01 jul 2021.

Câncer de testículo. Brasilia DF, 2021. Disponível em: < https://www.inca.gov.br/tipos-de-cancer/cancer-de-testiculo >. Acesso em: 06 mar 2021.

KIER, M. G. et al. Second Malignant Neoplasms and Cause of Death in Patients With Germ Cell Cancer. JAMA Oncology, v. 2, n. 12, p. 1624-1627, 2016.

KREGE, S. et al. European consensus conference on diagnosis and treatment of germ cell cancer: a report of the second meeting of the European Germ Cell Cancer Consensus group (EGCCCG): part I. Eur Urol v. 53, n. 3, p. 478-496, 2008.

LIP, S. Z. L. et al. A meta-analysis of the risk of boys with isolated cryptorchidism developing testicular cancer in later life. Archives of Disease in Childhood, v. 98, n. 1, p. 20-26, 2012.

MOUL, J. W. Diagnosis of Testicular Cancer. Urologic Clinics of North America, v. 34, n. 2, p. 109-117, 2007. 
NCl. Cancer Stat Facts: Testicular Cancer. USA, 2021. Disponível em: < https://seer.cancer.gov/statfacts/html/testis.html >. Acesso em: 03 mar 2021.

NERY, R. C. Câncer de Testículo. In: SANTOS, M. (Ed.). Diretrizes oncológicas 2. São Paulo SP: Doctor Press Ed. Científica, 2019.

PARK, J. S. et al. Recent global trends in testicular cancer incidence and mortality. Medicine, v. 97, n. 37, p. 1-7, 2018. Disponível em: < https://www.ncbi.nlm.nih.gov/pmc/articles/PMC6155960 >.

PIERORAZIO, P. M. et al. Non-risk-adapted Surveillance for Stage I Testicular Cancer: Critical Review and Summary. European Urology, v. 73, n. 6, p. 899907, 2018.

RAJPERT-DE-MEYTS, E. et al. Testicular germ cell tumours. The Lancet, v. 387, n. 10029, p. 1762-1774, 2016.

ROBINSON, $R$. et al. Is it safe to insert a testicular prosthesis at the time of radical orchidectomy for testis cancer: an audit of 904 men undergoing radical orchidectomy. BJU International, v. 117, n. 2, 2015.

ROSEN, A. et al. Global Trends in Testicular Cancer Incidence and Mortality. European Urology, v. 60, n. 2, p. 374-379, 2011.

SHAW, J. Diagnosis and Treatment of Testicular Cancer. American Family Physician, v. 7, n. 4, p. 469-474, 2008.

SMITH, Z. L.; WERNTZ, R. P.; EGGENER, S. E. Testicular Cancer: Epidemiology, Diagnosis, and Management. Medical Clinics of North America, v. 102, n. 2, p. 251264 , 2018. Disponível em: https://www.sciencedirect.com/science/article/abs/pii/S0025712517301578?via\%3Di hub $>$. 
WALSH, T. J. et al. Prepubertal Orchiopexy for Cryptorchidism May be Associated With Lower Risk of Testicular Cancer. The Journal of Urology, v. 178, n. 4, p. 1440 1446, 2007.

Enviado: Octubre de 2021.

Aprobado: Octubre de 2021. 